\title{
Optimal splitting of Parseval frames using Walsh matrices
}

\author{
AMIE ALBRECHT, PHIL HOWLETT AND GEETIKA VERMA ${ }^{\dagger}$
}

\author{
Date of Receiving : $\quad 11.03 .2018$ \\ Date of Revision : $\quad 01.04 .2018$ \\ Date of Acceptance : 21.06 .2018
}

\begin{abstract}
In 2014 Adam Marcus, Daniel Spielman and Nikhil Srivastava used random vectors to prove a key discrepancy theorem and in so doing gave a positive answer to the long-standing Kadison-Singer Problem. In this paper we use Walsh matrices to construct a class of natural frames in Euclidean space and discuss how these frames relate to the key discrepancy theorem.
\end{abstract}

\section{Introduction}

In 1959 Richard Kadison and Isadore Singer [11] formulated a problem in quantum mechanics that later became one of the iconic mathematical questions of the twentieth century. The problem is now known as the Kadison-Singer Problem (KSP).

Problem 1.1 (KSP). Does every pure state on the algebra of bounded diagonal operators acting on the Hilbert space of square summable complex-valued sequences have a unique extension to a regular state on the algebra of all bounded operators?

Following a finite-dimensional reformulation [2] by Joel Anderson in 1979 and further reduction to an equivalent problem in discrepancy theory [17] by Nik Weaver in 2004, a positive answer to KSP was eventually found [12, 13] by Adam Marcus, Daniel Spielman and Nikhil Srivastava in 2013. We will not attempt a detailed explanation of KSP but instead refer readers to the excellent review article [8] by Nick Harvey. The Marcus-Spielman-Srivistava Discrepancy Theorem (MSSDT) was a basic platform for the eventual solution of KSP and is a central theme in our paper.

2010 Mathematics Subject Classification. 11K38, 15B34, 42C10, 42C15.

Key words and phrases. Discrepancy theory, Walsh matrices, Walsh functions, frames in Euclidean space.

This research is funded by the Australian Research Council Discovery Grant DP 160101236 held by Phil Howlett, Amie Albrecht, Jerzy Filar and Konstantin Avrachenkov. Geetika Verma is employed by the project as a Research Associate. The authors would like to thank Dr Lalit Vashisht for helpful advice about preparation of the manuscript.

Communicated by: Ya. Farkov

${ }^{\dagger}$ Corresponding author. 